\title{
A New Adaptive Tracking Control Approach for Uncertain Flexible Joint Robot System
}

\author{
Zhen-Guo Liu ${ }^{1} \quad$ Jin-Ming Huang ${ }^{2}$ \\ ${ }^{1}$ School of Automation, Southeast University, Nanjing 210096, China \\ ${ }^{2}$ School of Engineering, Qufu Normal University, Qufu 273165, China
}

\begin{abstract}
The adaptive tracking problem for uncertain flexible joint robot system is studied in this paper. By utilizing the adaptive backstepping method, an adaptive controller is constructed at the beginning. By utilizing the modified adaptive dynamic surface control technique, a new adaptive controller is presented afterwards to avoid the overparametrization problem and the explosion of complexity problem existing in the adaptive backstepping method. All the signals of the closed-loop system are rendered globally/semi-globally uniformly ultimately bounded, and the tracking error can be made arbitrarily small by tuning the designed parameters. A simulation example is given to show the validity of the control algorithm.
\end{abstract}

Keywords: Flexible joint robots, adaptive control, backstepping method, dynamic surface control, position tracking.

\section{Introduction}

The research of flexible joint robots (FJR) has received considerable attention in the past two decades ${ }^{[1]}$. To obtain good control performance, no matter in modeling or in control design, the joint flexibility, which is usually caused by harmonic drives, shaft windup, and bearing deformation, cannot be ignored. In the literature, there have been many methods proposed, for instance, the singular perturbation approach ${ }^{[2]}$, the passivity approach ${ }^{[3]}$, the sliding mode approach $^{[4]}$, and the neural network approach ${ }^{[5,6]}$.

It is known that the backstepping technique is also an important method. A robust controller was proposed to guarantee the tracking of any given reference trajectory with arbitrary accuracy ${ }^{[7]}$. An adaptive output-feedback controller was designed for the single link robotic manipulator ${ }^{[8]}$ and the adaptive backstepping method for rigid-link flexiblejoint robots was studied. In addition to these methods ${ }^{[9,10]}$, the dynamic surface control method is a recently proposed control algorithm. With this method, Zhang et al. ${ }^{[11,12]}$ considered adaptive dynamic surface control for nonlinear systems with uncertainties, Hou and Duan ${ }^{[13]}$ studied how to design controller for integrated missile guidance and autopilot. Moreover, some other approaches such as model reference adaptive control ${ }^{[14,15]}$, robust adaptive control ${ }^{[16]}$ and adaptive iterative learning control ${ }^{[17]}$ are also useful to regulate such nonlinear systems.

The adaptive tracking problem for FJR has been studied before. However, there exist some drawbacks in the exist-

\footnotetext{
Regular Paper

Manuscript received November 14, 2013; accepted August 1, 2014

This work was supported by National Natural Science Foundation of China (No.61273091), the Project of Taishan Scholar of Shandong Province, and the Ph. D. Programs Foundation of Ministry of Education of China.

Recommended by Associate Editor Min Cheol Lee

(C) Institute of Automation, Chinese Academy of Science and Springer-Verlag Berlin Heidelberg 2015
}

ing methods. The first one may be the "overparametrization" problem. The reason is that the control design procedure depends on the linear property of unknown system parameters. Another may be the "explosion of complexity" problem, i.e., the designed controller is usually made quite complicated because of the repeated differentiations of virtual controllers in control design procedure. In this paper, we will investigate how to design an adaptive tracking controller for FJR to avoid the above problems. Mainly motivated by the continuous control ideas ${ }^{[18,19]}$ and flexibly using algebraic techniques, we present a new control design method for FJR. Then, we construct an appropriate Lyapunov function and show that the designed controllers can guarantee all the signals of the resulting closed-loop system globally/semi-globally uniformly ultimately bounded, and the tracking errors can be rendered arbitrarily small.

The main contributions of the paper are characterized by the following specific features: 1) The "explosion of complexity" problem and the "overparametrization" problem of the existing control methods are avoided. 2) It is not easy to find an appropriate Lyapunov function which is wellbehaved in stability analysis. In this paper, by using flexible algebraic techniques, two new Lyapunov functions are recursively constructed in the control design procedure.

\section{Preliminaries and problem statement}

Consider the dynamic equations of the flexible joint robots given as ${ }^{[20,21]}$

$$
\begin{aligned}
& M\left(q_{1}\right) \ddot{q}_{1}+C\left(q_{1}, \dot{q}_{1}\right) \dot{q}_{1}+h\left(q_{1}\right)+\tau_{c}+f_{1}\left(q_{1}, \dot{q}_{1}\right)=0 \\
& B \ddot{q}_{2}-\tau_{c}+f_{2}\left(q_{2}, \dot{q}_{2}\right)=u \\
& \tau_{c}=K\left(q_{1}-q_{2}\right)
\end{aligned}
$$

where $q_{1} \in \mathbf{R}^{n}$ is the joint angular position, $q_{2} \in \mathbf{R}^{n}$ is the motor angular position, $\dot{q}_{1}, \dot{q}_{2} \in \mathbf{R}^{n}$ are the respective veloc- 
ities, $M\left(q_{1}\right) \in \mathbf{R}^{n \times n}, B \in \mathbf{R}^{n \times n}$ are positive and symmetric inertia matrices, $K \in \mathbf{R}^{n \times n}$ is diagonal matrix whose entries are elastic constants $k_{i}$ of the joints, $C\left(q_{1}, \dot{q}_{1}\right) \dot{q}_{1} \in \mathbf{R}^{n}$ is the centrifugal and coriolis force, $h\left(q_{1}\right) \in \mathbf{R}^{n}$ is the gravity force vector, $f_{1}\left(q_{1}, \dot{q}_{1}\right), f_{2}\left(q_{2}, \dot{q}_{2}\right)$ are the frictional terms, and $u \in \mathbf{R}^{n}$ is the input torque. The angular positions $q_{1}$, $q_{2}$, and the respective velocities $\dot{q}_{1}, \dot{q}_{2}$ are assumed to be measurable.

The purpose of the paper is to design an adaptive state feedback controller for the system (1)-(3). We specified the control problems as: 1) Given the desired reference trajectory $q_{d}$, design a controller if possible, such that the link position tracking error $z_{1}=q_{1}-q_{d}$ converges to 0 as much as possible. 2) Meanwhile, all the closed-loop signals are rendered bounded.

We need Assumptions 1-3.

Assumption 1. The desired trajectory vectors are continuous and available, and $\left[q_{d}^{\mathrm{T}}, \dot{q}_{d}^{\mathrm{T}}, \ddot{q}_{d}^{\mathrm{T}}\right]^{\mathrm{T}} \in \Omega_{d}$ with known compact set $\Omega_{d}=\left\{\left.\left[q_{d}^{\mathrm{T}}, \dot{q}_{d}^{\mathrm{T}}, \ddot{q}_{d}^{\mathrm{T}}\right]^{\mathrm{T}} \in \mathbf{R}^{3 n}|| q_{d}\right|^{2}+\left|\dot{q}_{d}\right|^{2}+\left|\ddot{q}_{d}\right|^{2} \leq\right.$ A\}.

Assumption 2. There exist positive constants $b_{i}, m_{i}$, $k_{i}, \quad i=1,2$, such that $m_{1} \leq \lambda_{\min }(M) \leq\|M\|_{2} \leq$ $\lambda_{\max }(M) \leq m_{2}, b_{1} \leq \lambda_{\min }(B) \leq\|B\|_{2} \leq \lambda_{\max }(B) \leq b_{2}$, $k_{1} \leq \lambda_{\min }(K) \leq\|K\|_{2} \leq \lambda_{\max }(K) \leq k_{2}$, where only parameter $b_{2}$ is known.

Assumption 3. There exist unknown positive constant $\theta_{i}$, and known smooth functions $\phi_{i}(\cdot), i=0,1,2$, such that

$$
\begin{aligned}
& \left|C\left(q_{1}, \dot{q}_{1}\right) \dot{q}_{1}+h\left(q_{1}\right)+K q_{1}\right|^{2} \leq \theta_{0} \phi_{0}\left(q_{1}, \dot{q}_{1}\right) \\
& \left|f_{1}\left(q_{1}, \dot{q}_{1}\right)\right|^{2} \leq \theta_{1} \phi_{1}\left(q_{1}, \dot{q}_{1}\right) \\
& \left|f_{2}\left(q_{2}, \dot{q}_{2}\right)\right|^{2} \leq \theta_{2} \phi_{2}\left(q_{2}, \dot{q}_{2}\right) .
\end{aligned}
$$

Remark 1. These assumptions are reasonable and not stronger than the existing ones. Assumption 1 gives basic conditions using dynamic surface control method. Assumption 2 provides that only one constant $b_{2}$ is known in this paper, while in $[8,10]$, all the parameters $b_{i}, m_{i}, k_{i}, i=1,2$ were assumed to be known. Assumption 3 is similar to Assumption 2 in [19] for the rigid joint case.

Remark 2. It is easy to see that the FJR system is underactuated. Tracking control problems of underactuated systems are more difficult, since there are fewer inputs than degrees of freedom. In addition, when the system is suffered by external disturbances, only some semi-global results can be obtained, rather than achieving global results. See for instance, the FJR system ${ }^{[7,9]}$, the ships ${ }^{[22]}$, the wheeled inverted pendulums ${ }^{[23]}$, and more general underactuated system ${ }^{[24]}$.

Before the control design procedure, we define $x_{1}=q_{1}$, $x_{2}=\dot{q}_{1}, x_{3}=q_{2}, x_{4}=\dot{q}_{2}$, and introduce the following transformations:

$$
\begin{cases}z_{1}=x_{1}-q_{d}, & z_{2}=\dot{z}_{1}+\left(c_{1}+1\right) z_{1} \\ z_{3}=x_{3}-x_{3}^{*}, & z_{4}=x_{4}-x_{4}^{*}\end{cases}
$$

where $x_{i}^{*}$ is the virtual control to be determined later. Then, we can deduce that

$$
\left\{\begin{array}{l}
\dot{z}_{1}=z_{2}-\left(c_{1}+1\right) z_{1} \\
\dot{z}_{2}=M^{-1}\left(x_{1}\right) K x_{3}-F_{1} \\
\dot{z}_{3}=x_{4}-\frac{\partial x_{3}^{*}}{\partial \hat{\theta}}-F_{2} \\
\dot{z}_{4}=B^{-1}\left(u+K\left(x_{1}-x_{3}\right)-f_{2}\right)-\frac{\partial x_{4}^{*}}{\partial \hat{\theta}} \dot{\hat{\theta}}-F_{3}
\end{array}\right.
$$

where $F_{i}$ are defined as $F_{1}=M^{-1}\left(\mathrm{C} x_{2}+h+K x_{1}+f_{1}\right)+$ $\ddot{q}_{d}-\left(c_{1}+1\right) z_{2}+\left(c_{1}+1\right)^{2} z_{1}, F_{2}=\frac{\partial x_{3}^{*}}{\partial z_{1}^{1}} \dot{z}_{1}+\frac{\partial x_{3}^{*}}{\partial z_{2}^{\top}} \dot{z}_{2}+\frac{\partial x_{3}^{*}}{\partial q_{d}^{T}} \dot{q}_{d}+$ $\frac{\partial x_{3}^{*}}{\partial \dot{q}_{d}^{T}} \ddot{q}_{d}$, and $F_{3}=\frac{\partial x_{4}^{*}}{\partial z_{1}^{T}} \dot{z}_{1}+\frac{\partial x_{4}^{*}}{\partial z_{2}^{T}} \dot{z}_{2}+\frac{\partial x_{4}^{*}}{\partial z_{3}^{T}} \dot{z}_{3}+\frac{\partial x_{4}^{*}}{\partial q_{d}^{T}} \dot{q}_{d}+\frac{\partial x_{4}^{*}}{\partial \dot{q}_{d}^{T}} \ddot{q}_{d}$. Define positive parameters $a=\frac{m_{2}}{k_{1}^{2}}$ and $\theta=\frac{k_{2}^{2}}{a^{2} m_{1}^{2}} \times$ $\max \left\{\theta_{0}, \theta_{1}, m_{1}^{2}, k_{2}^{2}, a^{2} m_{1}^{2}, \frac{a m_{1}^{2}}{k_{2}}, \frac{\theta_{2} a^{2} m_{1}^{2}}{k_{2}^{2}}\right\}$. Parameter $a$ will be used for constructing the Lyapunov function subsequently, and parameter $\theta$ is the only one to be estimated in the control design. The following lemma 1, which is the well known Young's inequality ${ }^{[19]}$, will play a key role in proving the main results of this paper.

Lemma 1. For vectors $x, y \in \mathbf{R}^{n}$, and scalar positive numbers $\epsilon>0, p>0$, there holds

$$
x^{\mathrm{T}} y \leq \frac{\epsilon^{p}}{p}|x|^{p}+\frac{1}{q \epsilon^{p}}|y|^{q}
$$

where $q=\frac{p}{p-1}$.

Lemma 2. There exist smooth nonnegative functions $\psi_{i}, i=1, \cdots, 4$, and positive constant $\nu$ such that

$$
\begin{aligned}
& -z_{3}^{\mathrm{T}} F_{2} \leq\left(z_{3}^{\mathrm{T}} z_{3}\right)\left(\psi_{1} \theta+\psi_{2}\right)+\nu \\
& -z_{4}^{\mathrm{T}} F_{3} \leq\left(z_{4}^{\mathrm{T}} z_{4}\right)\left(\psi_{3} \theta+\psi_{4}\right)+\nu
\end{aligned}
$$

Proof. See Appendix.

\section{Control of FJR system}

In this subsection, we will construct an adaptive statefeedback controller for FJR system, which will be addressed in a step-by-step manner.

Step 1. Suppose $\hat{\theta}$ is the estimate of $\theta$, and the corresponding error is defined as $\tilde{\theta}=\hat{\theta}-\theta$. Then, we introduce the transformations: $z_{1}=x_{1}-q_{d}$ and $z_{2}=\dot{z}_{1}+\left(c_{1}+1\right) z_{1}$ and choose the candidate Lyapunov function $V_{1}\left(z_{1}, z_{2}, \tilde{\theta}\right)=$ $\frac{1}{2} z_{1}^{\mathrm{T}} z_{1}+\frac{1}{2 a} z_{2}^{\mathrm{T}} K z_{2}+\frac{1}{2 \gamma} \tilde{\theta}^{2}$, where $\gamma>0$ is a designed parameter. Taking the time derivative of $V_{1}$, we get

$$
\begin{gathered}
\dot{V}_{1}=-\left(c_{1}+1\right) z_{1}^{\mathrm{T}} z_{1}+z_{1}^{\mathrm{T}} z_{2}+\frac{1}{a} z_{2}^{\mathrm{T}} K M^{-1}\left(x_{1}\right) K x_{3}^{*}+ \\
\frac{1}{a} z_{2}^{\mathrm{T}} K M^{-1} K\left(x_{3}-x_{3}^{*}\right)-\frac{1}{a} z_{2}^{\mathrm{T}} K F_{1}+\frac{1}{\gamma} \tilde{\theta} \dot{\hat{\theta}}
\end{gathered}
$$

By using Lemma 1, one can conclude that

$$
z_{1}^{\mathrm{T}} z_{2} \leq \frac{1}{2} z_{1}^{\mathrm{T}} z_{1}+\frac{1}{2} z_{2}^{\mathrm{T}} z_{2}
$$


From Assumption 2 and Lemma 1, it leads to

$$
\begin{gathered}
-\frac{1}{a} z_{2}^{\mathrm{T}} K M^{-1}\left(C x_{2}+h\left(x_{1}\right)+K x_{1}+f_{1}\right) \leq \\
\frac{k_{2}}{a m_{1}}\left|z_{2}\right|\left|C x_{2}+h+K x_{1}+f_{1}\right| \leq \\
\frac{1}{\delta_{1}}\left(\frac{k_{2}}{a m_{1}}\right)^{2}\left|z_{2}\right|^{2}\left(\theta_{0} \phi_{0}+\theta_{1} \phi_{1}\right)+\frac{\delta_{1}}{2} \leq \\
\frac{1}{\delta_{1}} \theta z_{2}^{\mathrm{T}} z_{2}\left(\phi_{0}+\phi_{1}\right)+\frac{\delta_{1}}{2} .
\end{gathered}
$$

According to Assumption 2 and Lemma 1, it yields

$$
-\frac{1}{a} z_{2}^{\mathrm{T}} K \ddot{q}_{d} \leq \frac{\theta}{2 \delta_{2}} z_{2}^{\mathrm{T}} z_{2}+\frac{\delta_{2}}{2}\left|\ddot{q}_{d}\right|^{2} .
$$

Similarly, we have

$$
\begin{aligned}
& -\frac{1}{a} z_{2}^{\mathrm{T}} K\left(-\left(c_{1}+1\right) z_{2}+\left(c_{1}+1\right)^{2} z_{1}\right) \leq \\
& \left(c_{1}+1+\frac{\left(c_{1}+1\right)^{4}}{2}\right) \max \left\{\frac{k_{2}}{a}, \frac{k_{2}^{2}}{a^{2}}\right\}\left|z_{2}\right|^{2}+\frac{1}{2}\left|z_{1}\right|^{2} \leq \\
& \left(c_{1}+1+\frac{\left(c_{1}+1\right)^{4}}{2}\right) \theta z_{2}^{\mathrm{T}} z_{2}+\frac{1}{2} z_{1}^{\mathrm{T}} z_{1} .
\end{aligned}
$$

Combining (13)-(15), it follows that

$$
-\frac{1}{a} z_{2}^{\mathrm{T}} K F_{1} \leq \frac{1}{2} z_{1}^{\mathrm{T}} z_{1}+\theta\left(\omega_{1}-1\right) z_{2}^{\mathrm{T}} z_{2}+\frac{\delta_{1}}{2}+\frac{\delta_{2}}{2}\left|\ddot{q}_{d}\right|^{2}
$$

where $\omega_{1}=\frac{1}{\delta_{1}}\left(\phi_{0}+\phi_{1}\right)+\frac{1}{2 \delta_{2}}+c_{1}+\frac{\left(c_{1}+1\right)^{4}}{2}+2$. It follows from Assumption 2 and Lemma 1 that

$$
\frac{1}{a} z_{2}^{\mathrm{T}} K M^{-1} K\left(x_{3}-x_{3}^{*}\right) \leq \frac{1}{2} z_{3}^{\mathrm{T}} z_{3}+\theta z_{2}^{\mathrm{T}} z_{2} .
$$

Defining $d_{1}=\frac{\delta_{1}}{2}+\frac{\delta_{2}}{2}\left|\ddot{q}_{d}\right|^{2}$, and substituting (12)-(17) into (11), we obtain

$$
\begin{aligned}
\dot{V}_{1} \leq & -c_{1} z_{1}^{\mathrm{T}} z_{1}+\frac{1}{a} z_{2}^{\mathrm{T}} K M^{-1}\left(x_{1}\right) K x_{3}^{*}+\left(\frac{1}{2}+\omega_{1} \theta\right) z_{2}^{\mathrm{T}} z_{2}+ \\
& \frac{1}{2} z_{3}^{\mathrm{T}} z_{3}+d_{1}+\frac{1}{\gamma} \tilde{\theta} \dot{\hat{\theta}}
\end{aligned}
$$

We choose the first virtual control

$$
x_{3}^{*}\left(z_{1}, z_{2}, q_{d}, \dot{q}_{d}, \hat{\theta}\right)=-\left(c_{2}+\frac{1}{2}+\omega_{1} \hat{\theta}\right) z_{2}
$$

where $c_{2}>0$ is a design parameter. The adaptive law is designed as

$$
\dot{\hat{\theta}}=\Psi-\sigma \hat{\theta}
$$

where $\Psi \geq 0$ will be determined later. Actually, the adaptive law can guarantee $\hat{\theta}(t)>0$ for any positive initial value, i.e., if $\hat{\theta}\left(t_{0}\right)>0$. From $(20)$, we have

$$
\hat{\theta}(t)=\hat{\theta}\left(t_{0}\right) \mathrm{e}^{-\sigma\left(t-t_{0}\right)}+\int_{t_{0}}^{t} \mathrm{e}^{-\sigma(t-s)} \Psi(s) \mathrm{d} s>0 .
$$

In view of $a>0$ and $\hat{\theta}>0$, it follows that

$$
\frac{1}{a} z_{2}^{\mathrm{T}} K M^{-1}\left(x_{1}\right) K x_{3}^{*} \leq-\left(c_{2}+\frac{1}{2}+\omega_{1} \hat{\theta}\right) z_{2}^{\mathrm{T}} z_{2} .
$$

Substituting (21) into (18), we obtain

$$
\dot{V}_{1} \leq-\sum_{i=1}^{2} c_{i} z_{i}^{\mathrm{T}} z_{i}+\frac{1}{2} z_{3}^{\mathrm{T}} z_{3}+\frac{\tilde{\theta}}{\gamma}\left(\dot{\hat{\theta}}-\Psi_{1}\right)+d_{1}
$$

where $\Psi_{1}=\gamma \omega_{1} z_{2}^{\mathrm{T}} z_{2}$. This completes Step 1. It can be viewed as the initialization of the whole design procedure.

Step 2. Choosing the candidate Lyapunov function $V_{2}\left(z_{1}, z_{2}, z_{3}, \tilde{\theta}\right)=V_{1}+\frac{1}{2} z_{3}^{\mathrm{T}} z_{3}$, and taking the time derivative, it leads to

$$
\begin{aligned}
\dot{V}_{2} \leq & -\sum_{i=1}^{2} c_{i} z_{i}^{\mathrm{T}} z_{i}+\frac{1}{2} z_{3}^{\mathrm{T}} z_{3}+z_{3}^{\mathrm{T}} x_{4}^{*}+\frac{\tilde{\theta}}{\gamma}\left(\dot{\hat{\theta}}-\Psi_{1}\right)+ \\
& d_{1}-z_{3}^{\mathrm{T}} \frac{\partial x_{3}^{*}}{\partial \hat{\theta}} \dot{\hat{\theta}}+z_{3}^{\mathrm{T}}\left(x_{4}-x_{4}^{*}\right)-z_{3}^{\mathrm{T}} F_{2} .
\end{aligned}
$$

By Lemma 1, we can deduce that

$$
z_{3}^{\mathrm{T}}\left(x_{4}-x_{4}^{*}\right) \leq \frac{1}{2} z_{4}^{\mathrm{T}} z_{4}+z_{3}^{\mathrm{T}} z_{3} .
$$

Using Lemma 2, we have

$$
-z_{3}^{\mathrm{T}} F_{2} \leq\left(z_{3}^{\mathrm{T}} z_{3}\right)\left(\psi_{1} \theta+\psi_{2}\right)+\nu
$$

Defining $\Psi_{2}=\Psi_{1}+\gamma \psi_{1} z_{3}^{\mathrm{T}} z_{3}$ and choosing the virtual control, we have

$$
x_{4}^{*}(\cdot)=-\left(c_{3}+1+\psi_{2}+\psi_{1} \hat{\theta}\right) z_{3}-\frac{\partial x_{3}^{*}}{\partial \hat{\theta}}\left(\Psi_{2}-\sigma \hat{\theta}\right)
$$

where $c_{3}>0$ is a design parameter. Substituting (26) into (23), it yields that

$$
\begin{gathered}
\dot{V}_{2} \leq-\sum_{i=1}^{3} c_{i} z_{i}^{\mathrm{T}} z_{i}+\frac{1}{2} z_{4}^{\mathrm{T}} z_{4}+\left(\frac{\tilde{\theta}}{\gamma}-z_{3}^{\mathrm{T}} \frac{\partial x_{3}^{*}}{\partial \hat{\theta}}\right) \times \\
\left(\dot{\hat{\theta}}-\Psi_{2}+\sigma \hat{\theta}\right)-\frac{\sigma}{\gamma} \tilde{\theta} \hat{\theta}+\mathrm{d}_{2}
\end{gathered}
$$

where $d_{2}=d_{1}+\nu$ is a positive constant.

Step 3. Choosing the candidate Lyapunov function as $V_{3}\left(z_{1}, z_{2}, z_{3}, z_{4}, \varrho_{1}, \varrho_{2}, \tilde{\theta}\right)=V_{2}+\frac{1}{2} z_{4}^{\mathrm{T}} B z_{4}$, and taking the time derivative of $V_{3}$ while noticing (7) and (8), we have

$$
\begin{gathered}
\dot{V}_{3} \leq-\sum_{i=1}^{3} c_{i} z_{i}^{\mathrm{T}} z_{i}+\frac{1}{2} z_{4}^{\mathrm{T}} z_{4}+\left(\frac{\tilde{\theta}}{\gamma}-z_{3}^{\mathrm{T}} \frac{\partial x_{3}^{*}}{\partial \hat{\theta}}\right) \times \\
\left(\dot{\hat{\theta}}-\Psi_{2}-\sigma \hat{\theta}\right)+\frac{\sigma}{\gamma} \tilde{\theta} \hat{\theta}+z_{4}^{\mathrm{T}} u-z_{4}^{\mathrm{T}} \frac{\partial x_{4}^{*}}{\partial \hat{\theta}} \dot{\hat{\theta}}+ \\
z_{4}^{\mathrm{T}}\left(K\left(x_{1}-x_{3}\right)-f_{2}\right)-z_{4}^{\mathrm{T}} F_{3}+d_{2} .
\end{gathered}
$$

By using Lemma 1, it leads to

$$
z_{4}^{\mathrm{T}} K\left(x_{1}-x_{3}\right) \leq \frac{\theta}{2 \epsilon_{1}}\left|z_{4}\right|^{2}\left|x_{1}-x_{3}\right|^{2}+\frac{\epsilon_{1}}{2} .
$$

Similarly, from Lemma 1, we can deduce that

$$
-z_{4}^{\mathrm{T}} f_{2} \leq \frac{\theta_{2}}{2 \epsilon_{2}}\left|z_{4}\right|^{2} \phi_{2}+\frac{\epsilon_{2}}{2} \leq \frac{\theta}{2 \epsilon_{2}}\left|z_{4}\right|^{2} \phi_{2}+\frac{\epsilon_{2}}{2} .
$$

By Lemma 2, it yields

$$
-z_{4}^{\mathrm{T}} F_{3} \leq\left(z_{4}^{\mathrm{T}} z_{4}\right)\left(\psi_{3} \theta+\psi_{4}\right)+\nu .
$$


Defining $\omega_{2}=\frac{1}{2 \epsilon_{1}}\left|x_{1}-x_{3}\right|^{2}+\frac{1}{2 \epsilon_{2}} \phi_{2}+\psi_{3}, \bar{\psi}_{3}=\psi_{3}-$ $\gamma \psi_{3} z_{3}^{\mathrm{T}} \frac{\partial x_{3}^{*}}{\partial \theta}, \Psi=\Psi_{2}+\gamma \omega_{2} z_{4}^{\mathrm{T}} z_{4}, d_{3}=d_{2}+\nu+\frac{\epsilon_{1}}{2}+\frac{\epsilon_{2}}{2}$, and choosing the adaptive controller, we have

$$
\left\{\begin{array}{l}
u=-\left(c_{4}+\frac{1}{2}+\bar{\psi}_{3}+\omega_{2} \hat{\theta}\right) z_{4}-\frac{\partial x_{4}^{*}}{\partial \hat{\theta}}(\Psi-\sigma \hat{\theta}) \\
\dot{\hat{\theta}}=\Psi-\sigma \hat{\theta}, \hat{\theta}(0)>0 .
\end{array}\right.
$$

Substituting (24) and (31) into (28), we have

$$
\dot{V}_{3} \leq-\sum_{i=1}^{3} c_{i} z_{i}^{\mathrm{T}} z_{i}+\frac{1}{2} z_{4}^{\mathrm{T}} z_{4}+\frac{\sigma}{\gamma} \tilde{\theta} \hat{\theta}+d_{3} .
$$

It is not difficult to get

$$
-\frac{1}{\gamma} \sigma \tilde{\theta} \hat{\theta}=-\frac{1}{\gamma} \sigma \tilde{\theta}^{2}-\frac{1}{\gamma} \sigma \tilde{\theta} \theta \leq-\frac{1}{2 \gamma} \sigma \tilde{\theta}^{2}+\frac{1}{2 \gamma} \sigma \theta^{2} .
$$

Substituting (34) into (33), it follows that

$$
\dot{V}_{3} \leq-c V_{3}+d
$$

where $d=d_{3}+\frac{1}{2 \gamma} \sigma \theta^{2}$. Theorem 1 summarizes the main results of this section.

Theorem 1. Consider the flexible joint robots dynamic system (1)-(3) under Assumptions 1-3, we can design the adaptive controller (32), such that all the closed-loop signals are rendered globally uniformly ultimately bounded and the tracking error $z_{1}$ can be rendered arbitrarily small.

Proof. As can be seen, by (35), we can deduce that $\dot{V}_{3} \leq 0$ on $V_{3}=\rho$ when $c>\frac{d}{\rho}$. Hence, $V_{3} \leq \rho$ is an invariant set, i.e., if $V_{3}(0) \leq \rho$, then $V_{3}(t) \leq \rho$ for all $t \geq 0$. Thus, all the closed-loop signals are globally uniformly ultimately bounded. Moreover, by adjusting parameters $\sigma, \gamma, c_{i}, \delta_{j}$, $\epsilon_{j}, i=1, \cdots, 4, j=1,2,3$, we can make the tracking error $z_{1}$ arbitrarily small.

Remark 3. This section presents an adaptive backstepping based control algorithm for FJR system. The overparametrization problem is avoided. However, the designed controller is very complicated here. This problem can be solved by using a modified adaptive dynamic surface method, see the next section for detail.

\section{Extensions}

In this section, a modified adaptive dynamic surface method will be adopted to obtain a simple adaptive controller. We need to introduce the following transformations:

$$
\begin{cases}\bar{z}_{1}=x_{1}-q_{d}, & \bar{z}_{2}=\dot{\bar{z}}_{1}+\left(\bar{c}_{1}+1\right) \bar{z}_{1} \\ \bar{z}_{3}=x_{3}-\bar{x}_{3}, & \bar{z}_{4}=x_{4}-\bar{x}_{4}\end{cases}
$$

where $\bar{x}_{i}, i=3,4$ are the filtered virtual control achieved by the following first-order filter

$$
\tau_{i} \dot{\bar{x}}_{i}+\bar{x}_{i}=x_{i}^{*}, \quad \bar{x}_{i}(0)=x_{i}^{*}(0)
$$

where $\tau_{i}>0$ is a positive constant and $x_{i}^{*}$ is the virtual control to be determined later. Define $\varrho_{1}=\bar{x}_{3}-x_{3}^{*}, \varrho_{2}=$ $\bar{x}_{4}-x_{4}^{*}$, then from (36) and (37), we have

$$
\left\{\begin{array}{l}
\dot{\bar{z}}_{1}=z_{2}-\left(\bar{c}_{1}+1\right) z_{1} \\
\dot{\bar{z}}_{2}=M^{-1}\left(x_{1}\right) K x_{3}-F\left(x_{1}, x_{2}, q_{d}, \dot{q}_{d}, \ddot{q}_{d}\right) \\
\dot{\bar{z}}_{3}=x_{4}+\frac{1}{\tau_{3}} \varrho_{1} \\
\dot{\bar{z}}_{4}=B^{-1}\left(u+K\left(x_{1}-x_{3}\right)-f_{2}\left(x_{3}, x_{4}\right)\right)+\frac{1}{\tau_{4}} \varrho_{2}
\end{array}\right.
$$

where $F$ is defined as

$$
\begin{aligned}
F= & M^{-1}\left(C x_{2}+h+K x_{1}+f_{1}\right)+\ddot{q}_{d}- \\
& \left(\bar{c}_{1}+1\right) \bar{z}_{2}+\left(\bar{c}_{1}+1\right)^{2} \bar{z}_{1} .
\end{aligned}
$$

Now, we give the design procedure in detail.

Step 1. Choosing the candidate Lyapunov function $U_{1}\left(\bar{z}_{1}, \bar{z}_{2}, \tilde{\theta}\right)=\frac{1}{2} \bar{z}_{1}^{\mathrm{T}} \bar{z}_{1}+\frac{1}{2 a} \bar{z}_{2}^{\mathrm{T}} K \bar{z}_{2}+\frac{1}{2 \bar{\gamma}} \tilde{\theta}^{2}$, similar to Section 3 , we have

$$
-\frac{1}{a} \bar{z}_{2}^{\mathrm{T}} K F \leq \frac{1}{2} \bar{z}_{1}^{\mathrm{T}} \bar{z}_{1}+\theta\left(\omega_{1}-1\right) \bar{z}_{2}^{\mathrm{T}} \bar{z}_{2}+\frac{\bar{\delta}_{1}}{2}+\frac{\bar{\delta}_{2}}{2}\left|\ddot{q}_{d}\right|^{2} .
$$

According to Assumption 2, Lemma 1 and noticing $x_{3}-$ $x_{3}^{*}=\bar{z}_{3}+\varrho_{1}$, it follows that

$$
\frac{1}{a} \bar{z}_{2}^{\mathrm{T}} K M^{-1} K\left(x_{3}-x_{3}^{*}\right) \leq \frac{1}{2} \bar{z}_{3}^{\mathrm{T}} \bar{z}_{3}+\frac{1}{2} \varrho_{1}^{\mathrm{T}} \varrho_{1}+\theta \bar{z}_{2}^{\mathrm{T}} \bar{z}_{2} .
$$

Choosing the virtual control

$$
x_{3}^{*}\left(z_{1}, z_{2}, q_{d}, \dot{q}_{d}, \hat{\theta}\right)=-\left(\bar{c}_{2}+\frac{1}{2}+\omega_{1} \hat{\theta}\right) z_{2}
$$

where $\bar{c}_{2}>0$ is a design parameter, and using (39) and (40), we have

$$
\dot{U}_{1} \leq-\sum_{i=1}^{2} \bar{c}_{i} \bar{z}_{i}^{\mathrm{T}} \bar{z}_{i}+\frac{1}{2} \bar{z}_{3}^{\mathrm{T}} \bar{z}_{3}+\frac{1}{2} \varrho_{1}^{\mathrm{T}} \varrho_{1}+\frac{\tilde{\theta}}{\bar{\gamma}}\left(\dot{\hat{\theta}}-\Lambda_{1}\right)+d_{1}
$$

where $\Lambda_{1}=\bar{\gamma} \omega_{1} \bar{z}_{2}^{\mathrm{T}} \bar{z}_{2}$. Let $x_{3}^{*}$ pass the first-order filter (37), then we get the filtered virtual control $\bar{x}_{3}$.

Step 2. Choosing the candidate Lyapunov function $U_{2}\left(z_{1}, z_{2}, \bar{z}_{3}, \tilde{\theta}\right)=U_{1}+\frac{1}{2} \bar{z}_{3}^{\mathrm{T}} \bar{z}_{3}$ and taking the time derivative, we have

$$
\begin{gathered}
\dot{U}_{2} \leq-\sum_{i=1}^{2} \bar{c}_{i} z_{i}^{\mathrm{T}} z_{i}+\frac{1}{2} \bar{z}_{3}^{\mathrm{T}} \bar{z}_{3}+\frac{1}{2} \varrho_{1}^{\mathrm{T}} \varrho_{1}+\frac{\tilde{\theta}}{\bar{\gamma}}\left(\dot{\hat{\theta}}-\Lambda_{1}\right)+ \\
\bar{z}_{3}^{\mathrm{T}}\left(x_{4}-x_{4}^{*}\right)+\bar{z}_{3}^{\mathrm{T}}\left(x_{4}^{*}+\frac{1}{\tau_{3}} \varrho_{1}\right)+d_{1} .
\end{gathered}
$$

From Lemma 1, it yields that

$$
\bar{z}_{3}^{\mathrm{T}}\left(x_{4}-x_{4}^{*}\right)=\bar{z}_{3}^{\mathrm{T}}\left(\bar{z}_{4}+\varrho_{2}\right) \leq \frac{1}{2} \bar{z}_{4}^{\mathrm{T}} \bar{z}_{4}+\bar{z}_{3}^{\mathrm{T}} \bar{z}_{3}+\frac{1}{2} \varrho_{2}^{\mathrm{T}} \varrho_{2} .
$$

Choose the virtual control

$$
x_{4}^{*}\left(\bar{z}_{3}, \varrho_{1}\right)=-\left(\frac{3}{2}+\bar{c}_{3}\right) \bar{z}_{3}-\frac{1}{\tau_{3}} \varrho_{1}
$$


where $\bar{c}_{3}>0$ is a designed parameter. Defining $\bar{d}_{1}=\frac{\bar{\delta}_{1}}{2}+$ $\frac{\bar{\delta}_{2}}{2}\left|\ddot{q}_{d}\right|^{2}$, and substituting (45) into (43), it yields that

$$
\begin{aligned}
\dot{U}_{2} \leq & -\sum_{i=1}^{3} \bar{c}_{i} \bar{z}_{i}^{\mathrm{T}} \bar{z}_{i}+\frac{1}{2} \bar{z}_{4}^{\mathrm{T}} \bar{z}_{4}+\frac{1}{2} \varrho_{1}^{\mathrm{T}} \varrho_{1}+\frac{1}{2} \varrho_{2}^{\mathrm{T}} \varrho_{2}+ \\
& \frac{\tilde{\theta}}{\gamma}\left(\dot{\hat{\theta}}-\Lambda_{1}\right)+\bar{d}_{1} .
\end{aligned}
$$

Let the virtual control $x_{4}^{*}$ pass the first-order filter (37), then we can get the filtered virtual control $\bar{x}_{4}$.

Step 3. From the definition of $\varrho_{1}, \varrho_{2}$, it follows that

$$
\left\{\begin{array}{l}
\dot{\varrho}_{1}=-\frac{1}{\tau_{3}} \varrho_{1}+\eta_{1}\left(\bar{z}_{1}, \cdots, \bar{z}_{4}, \varrho_{1}, q_{d}, \dot{q}_{d}, \ddot{q}_{d}, \hat{\theta}\right) \\
\varrho_{2}=-\frac{1}{\tau_{4}} \varrho_{2}+\eta_{2}\left(\bar{z}_{1}, \cdots, \bar{z}_{4}, \varrho_{1}, \varrho_{2}, q_{d}, \dot{q}_{d}, \ddot{q}_{d}, \hat{\theta}\right)
\end{array}\right.
$$

where continuous functions $\eta_{1}, \eta_{2}$ are defined as

$$
\begin{aligned}
& \eta_{1}=\frac{\partial x_{3}^{*}}{\partial \bar{z}_{1}^{\mathrm{T}}} \dot{\bar{z}}_{1}+\frac{\partial x_{3}^{*}}{\partial \bar{z}_{2}^{\mathrm{T}}} \dot{\bar{z}}_{2}+\frac{\partial x_{3}^{*}}{\partial q_{d}^{\mathrm{T}}} \dot{q}_{d}+\frac{\partial x_{3}^{*}}{\partial \dot{q}_{d}^{\mathrm{T}}} \ddot{q}_{d}+\frac{\partial x_{3}^{*}}{\partial \hat{\theta}} \dot{\hat{\theta}} \\
& \eta_{2}=\frac{\partial x_{4}^{*}}{\partial \bar{z}_{3}^{\mathrm{T}}} \dot{\bar{z}}_{3}+\frac{\partial x_{4}^{*}}{\partial \varrho_{1}^{\mathrm{T}}} \dot{\varrho}_{1} .
\end{aligned}
$$

Choosing the candidate Lyapunov function $U_{3}\left(\bar{z}_{1}, \bar{z}_{2}, \bar{z}_{3}, \bar{z}_{4}, \varrho_{1}, \varrho_{2}, \tilde{\theta}\right)=U_{2}+\frac{1}{2} \bar{z}_{4}^{\mathrm{T}} B \bar{z}_{4}+\frac{1}{2} \varrho_{1}^{\mathrm{T}} \varrho_{1}+\frac{1}{2} \varrho_{2}^{\mathrm{T}} \varrho_{2}$, and taking the time derivative of $V_{3}$ while noticing (36) and (38), we have

$$
\begin{aligned}
\dot{U}_{3} \leq & -\sum_{i=1}^{3} \bar{c}_{i} \bar{z}_{i}^{\mathrm{T}} \bar{z}_{i}+\frac{1}{2} \bar{z}_{4}^{\mathrm{T}} \bar{z}_{4}+\frac{1}{2} \varrho_{1}^{\mathrm{T}} \varrho_{1}+\frac{1}{2} \varrho_{2}^{\mathrm{T}} \varrho_{2}+\bar{d}_{1}+ \\
& \frac{\tilde{\theta}}{\bar{\gamma}}\left(\dot{\hat{\theta}}-\Lambda_{1}\right)+\bar{z}_{4}^{\mathrm{T}} u-\varrho_{1}^{\mathrm{T}}\left(\frac{1}{\tau_{3}} \varrho_{1}-\eta_{1}\right)-\varrho_{2}^{\mathrm{T}}\left(\frac{1}{\tau_{4}} \varrho_{2}-\eta_{2}\right)+ \\
& \bar{z}_{4}^{\mathrm{T}}\left(K\left(x_{1}-x_{3}\right)-f_{2}+\frac{1}{\tau_{4}} B \varrho_{2}\right) .
\end{aligned}
$$

There exist positive constants $\bar{\epsilon}_{1}$ and $\bar{\epsilon}_{2}$ such that

$$
\bar{z}_{4}^{\mathrm{T}}\left(K\left(x_{1}-x_{3}\right)-f_{2}\right) \leq \theta \omega_{2} \bar{z}_{4}^{\mathrm{T}} \bar{z}_{4}+\frac{1}{2} \varrho_{2}^{\mathrm{T}} \varrho_{2}+\frac{\bar{\epsilon}_{1}}{2}+\frac{\bar{\epsilon}_{2}}{2}
$$

where $\omega_{2}$ is defined as $\omega_{2}=\frac{1}{2 \bar{\epsilon}_{1}}\left|x_{1}-x_{3}\right|^{2}+\frac{1}{2 \bar{\epsilon}_{2}} \phi_{2}$. By using Lemma 1 , we can deduce

$$
\bar{z}_{4}^{\mathrm{T}} \times \frac{1}{\tau_{4}} B \varrho_{2} \leq \frac{1}{\bar{\epsilon}_{3} \tau_{4}^{2}}\left|\bar{z}_{4}\right|^{2}+\frac{\bar{\epsilon}_{3} b_{2}^{2}}{4} \varrho_{2}^{2}
$$

Defining $\bar{d}_{2}=\bar{d}_{1}+\frac{\bar{\epsilon}_{1}}{2}+\frac{\bar{\epsilon}_{2}}{2}$, and substituting (52) and (51) into (50), we have

$$
\begin{gathered}
\dot{U}_{3} \leq-\sum_{i=1}^{3} \bar{c}_{i} \bar{z}_{i}^{\mathrm{T}} \bar{z}_{i}+\frac{\tilde{\theta}}{\bar{\gamma}}(\dot{\hat{\theta}}-\Lambda)+\bar{z}_{4}^{\mathrm{T}} u+\varrho_{1}^{\mathrm{T}} \eta_{1}+\varrho_{2}^{\mathrm{T}} \eta_{2}+ \\
\left(\frac{1}{2}+\frac{1}{\bar{\epsilon}_{3} \tau_{4}^{2}}+\omega_{2} \hat{\theta}\right) \bar{z}_{4}^{\mathrm{T}} \bar{z}_{4}+\left(-\frac{1}{\tau_{3}}+\frac{1}{2}\right) \varrho_{1}^{\mathrm{T}} \varrho_{1}+ \\
\left(-\frac{1}{\tau_{4}}+1+\frac{\bar{\epsilon}_{3} b_{2}^{2}}{4}\right) \varrho_{2}^{\mathrm{T}} \varrho_{2}+\bar{d}_{2}
\end{gathered}
$$

where $\Lambda=\Lambda_{1}+\bar{\gamma} \omega_{2} \bar{z}_{4}^{\mathrm{T}} \bar{z}_{4}$. Choosing the adaptive controller

$$
\left\{\begin{array}{l}
u=-\left(\frac{1}{2}+\frac{1}{\bar{\epsilon}_{3} \tau_{4}^{2}}+\bar{c}_{4}+\omega_{2} \hat{\theta}\right) \bar{z}_{4} \\
\dot{\hat{\theta}}=\Lambda-\bar{\sigma} \hat{\theta}, \hat{\theta}(0)>0
\end{array}\right.
$$

where $\bar{\sigma}$ is a positive constant, and substituting (54) into (53), it leads to

$$
\begin{aligned}
\dot{U}_{3} \leq & -\sum_{i=1}^{4} \bar{c}_{i} \bar{z}_{i}^{\mathrm{T}} \bar{z}_{i}-\frac{1}{\bar{\gamma}} \bar{\sigma} \tilde{\theta} \hat{\theta}+\left(-\frac{1}{\tau_{3}}+\frac{1}{2}\right) \varrho_{1}^{\mathrm{T}} \varrho_{1}+ \\
& \left(-\frac{1}{\tau_{4}}+1+\frac{\epsilon_{3} b_{2}^{2}}{4}\right) \varrho_{2}^{\mathrm{T}} \varrho_{2}+\varrho_{1}^{\mathrm{T}} \eta_{1}+\varrho_{2}^{\mathrm{T}} \eta_{2}+d_{2} .
\end{aligned}
$$

Now, we have theorem 2, which summarizes the main results of this section.

Theorem 2. Consider the flexible joint robots dynamic system (1) - (3) under Assumptions 1-3, one can design the adaptive state-feedback controller (54), such that:

1) For any initial conditions satisfying $U_{3}(0) \leq \rho, \rho>0$, there exist $\sigma, \bar{\gamma}, \bar{c}_{i}, \bar{\delta}_{j}, \bar{\epsilon}_{j}, \tau_{k}, i=1, \cdots, 4, j=1,2,3, k=$ 3,4 , guaranteeing that the tracking error $z_{1}$ can be made arbitrarily small by adjusting these designed parameters.

2) All the closed-loop signals are rendered semi-globally uniformly ultimately bounded.

Proof. Choosing the Lyapunov function $V_{3}$, and defining the set $\Omega=\left\{\left[\bar{z}_{1}^{\mathrm{T}}, \bar{z}_{2}^{\mathrm{T}}, \bar{z}_{3}^{\mathrm{T}}, \bar{z}_{4}^{\mathrm{T}}, \varrho_{1}^{\mathrm{T}}, \varrho_{2}^{\mathrm{T}}, \tilde{\theta}\right]^{\mathrm{T}} \in \mathbf{R}^{6 n+1} \mid \bar{z}_{1}^{\mathrm{T}} \bar{z}_{1}+\right.$ $\left.\frac{1}{a} \bar{z}_{2}^{\mathrm{T}} K \bar{z}_{2}+\bar{z}_{3}^{\mathrm{T}} \bar{z}_{3}+\bar{z}_{4}^{\mathrm{T}} B \bar{z}_{4}+\varrho_{1}^{\mathrm{T}} \varrho_{1}+\varrho_{2}^{\mathrm{T}} \varrho_{2}+\frac{1}{\bar{\gamma}} \tilde{\theta}^{2} \leq 2 \rho\right\}$, we see that $\Omega_{d}$ in Assumption 1 and $\Omega$ are compact sets. Then, from the definition of $\eta_{1}(\cdot)$ and $\eta_{2}(\cdot)$, it follows that there exist positive constants $\bar{\eta}_{1}$ and $\bar{\eta}_{2}$, such that on the compact set $\Omega_{d} \times \Omega,\left|\eta_{1}(\cdot)\right| \leq \bar{\eta}_{1}$ and $\left|\eta_{2}(\cdot)\right| \leq \bar{\eta}_{2}$. By using Lemma 1 , we have

$$
\varrho_{1}^{\mathrm{T}} \eta_{1} \leq \frac{\bar{\eta}_{1}^{2}}{2 \bar{\delta}_{3}} \varrho_{1}^{\mathrm{T}} \varrho_{1}+\frac{\bar{\delta}_{3}}{2}, \quad \varrho_{2}^{\mathrm{T}} \eta_{2} \leq \frac{\bar{\eta}_{2}^{2}}{2 \bar{\delta}_{4}} \varrho_{2}^{\mathrm{T}} \varrho_{2}+\frac{\bar{\delta}_{4}}{2} .
$$

Defining $\bar{d}=\bar{d}_{2}+\frac{\bar{\delta}_{3}}{2}+\frac{\bar{\delta}_{4}}{2}+\frac{1}{2 \bar{\gamma}} \bar{\sigma} \theta^{2}$, and substituting (34) and (56) into (55) yields

$$
\begin{gathered}
\dot{U}_{3} \leq \\
-\sum_{i=1}^{4} \bar{c}_{i} \bar{z}_{i}^{\mathrm{T}} \bar{z}_{i}-\frac{1}{2 \bar{\gamma}} \bar{\sigma} \tilde{\theta}^{2}-\left(\frac{1}{\tau_{3}}-\frac{1}{2}-\frac{\bar{\eta}_{1}^{2}}{2 \bar{\delta}_{3}}\right) \varrho_{1}^{\mathrm{T}} \varrho_{1}- \\
\left(\frac{1}{\tau_{4}}-1-\frac{\bar{\epsilon}_{3} b_{2}^{2}}{4}-\frac{\bar{\eta}_{2}^{2}}{2 \bar{\delta}_{4}}\right) \varrho_{2}^{\mathrm{T}} \varrho_{2}+\bar{d}
\end{gathered}
$$

Choosing parameters such that $\bar{c}_{i}>0, i=1, \cdots, 4, \bar{\sigma}>0$, $\frac{1}{\tau_{3}} \geq \tau_{3}^{*}+\frac{1}{2}+\frac{\bar{\eta}_{1}^{2}}{2 \delta_{3}}, \frac{1}{\tau_{4}} \geq \tau_{4}^{*}+1+\frac{\bar{\epsilon}_{3} b_{2}^{2}}{4}+\frac{\bar{\eta}_{2}^{2}}{2 \delta_{4}}$, where $\tau_{3}^{*}>0$, $\tau_{4}^{*}>0$, we have

$$
\dot{U}_{3} \leq-c U_{3}+\bar{d}
$$

where $c$ satisfies $0<\bar{c} \leq 2 \cdot \min \left\{\bar{c}_{1}, \frac{a \bar{c}_{2}}{k_{2}}, \bar{c}_{3}, \frac{\bar{c}_{4}}{b_{2}}, \frac{1}{2} \bar{\sigma}, \tau_{3}^{*}, \tau_{4}^{*}\right\}$. It follows from (58) that $\dot{U}_{3} \leq 0$ on the surface $U_{3}=\rho$ when $\bar{c}>\frac{d}{\rho}$. Hence, $V_{3} \leq \rho$ is an invariant set, i.e., if $U_{3}(0) \leq$ $\rho$, then $U_{3}(t) \leq \rho$ for all $t \geq 0$. Consequently, all the closed-loop signals are semi-globally uniformly ultimately bounded. Moreover, by adjusting parameters $\bar{\sigma}, \bar{\gamma}, \bar{c}_{i}, \bar{\delta}_{j}$, $\bar{\epsilon}_{j}, \tau_{k}, i=1, \cdots, 4, j=1,2,3, k=3,4$, we can make the tracking error $\bar{z}_{1}$ arbitrarily small.

Remark 4. To achieve the tracking errors arbitrarily small, one can adjust the design parameters accordingly. To be specific, increasing $\bar{c}_{i}, \bar{\sigma}$, and decreasing $\tau_{k}$ will help to increase $\bar{c}$. Decreasing $\bar{\delta}_{j}, \bar{\epsilon}_{j}$ and increasing $\bar{\gamma}$ will help to decrease $\bar{d}$. From (58), it leads to $U_{3} \leq U_{3}(0) \mathrm{e}^{-\bar{c} t}+\frac{\bar{d}}{\bar{c}}(1-$ $\left.\mathrm{e}^{-\bar{c} t}\right)$. With increasing value of $\bar{c}$, and decreasing value of $\frac{\bar{d}}{\bar{c}}$, the tracking error is made smaller. 


\section{Simulation}

Consider the single-link flexible joint robot $^{[7,9]}$, whose dynamic equations are as follows:

$$
\begin{aligned}
& I \ddot{q}_{1}+M g l \sin \left(q_{1}\right)+K\left(q_{1}-q_{2}\right)+f_{1}\left(q_{1}, \dot{q}_{1}\right)=0 \\
& J \ddot{q}_{2}+B \dot{q}_{2}+K\left(q_{2}-q_{1}\right)+f_{2}\left(q_{2}, \dot{q}_{2}\right)=u .
\end{aligned}
$$

The desired trajectory for this robot model is given as $q_{d}=0.5 \sin (0.5 t) \mathrm{rad}$. When the external disturbances are 0 , we choose the parameters as $I=1 \mathrm{~kg} \cdot \mathrm{m}^{2}, M g l=3 \mathrm{~N} \cdot \mathrm{m}$, $K=5 \mathrm{~N} \cdot \mathrm{m} / \mathrm{rad}, J=1 \mathrm{~kg} \cdot \mathrm{m}^{2}, B=4 \mathrm{~N} \cdot \mathrm{m} \cdot \mathrm{s} / \mathrm{rad}$. It is easy to verify that Assumption 1 and Assumption 2 hold. Assumption 3 holds with $\phi_{0}=\sin ^{2} q_{1}+q_{1}^{2}, \phi_{1}=0, \phi_{2}=\dot{q}_{2}^{2}$. In Figs. 1-3, the responses of the resulting closed-loop system for this case are characterized by the different curves (the position of the link is $q_{1 a}$, the parameter estimation is $\theta_{a}$, and the control input is $\left.u_{a}\right)$. When the external disturbances are $f_{1}=-0.5 \cos \left(q_{1}\right) \mathrm{N}$ and $f_{2}=-0.5 \cos \left(q_{2}\right) \mathrm{N}$, we choose another group of the parameters as $I=0.5 \mathrm{~kg} \cdot \mathrm{m}^{2}$, $M g l=1.5 \mathrm{~N} \cdot \mathrm{m}, K=2.5 \mathrm{~N} \cdot \mathrm{m} / \mathrm{rad}, J=0.5 \mathrm{~kg} \cdot \mathrm{m}^{2}$, and $B=2 \mathrm{~N} \cdot \mathrm{m} \cdot \mathrm{s} / \mathrm{rad}$, the responses of the resulting closed-loop system for this case are characterized by the different curves (the position of the link is $q_{1 b}$, the parameter estimation is $\theta_{b}$, and the control input is $u_{b}$ ).

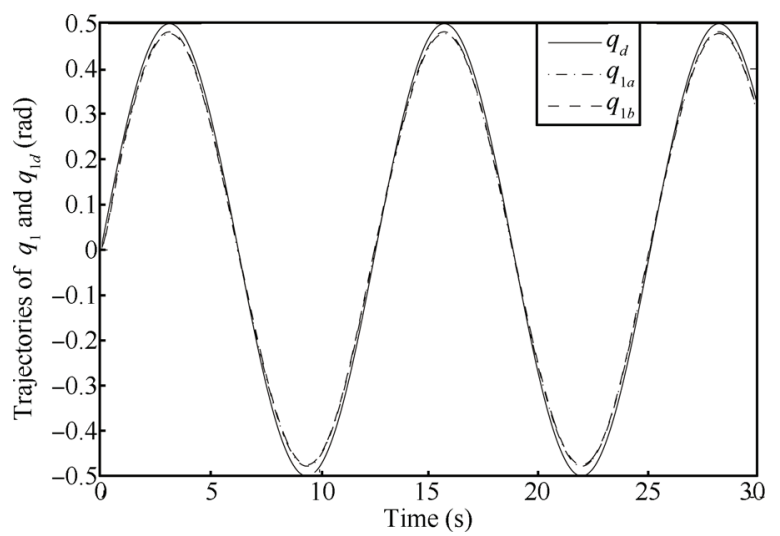

Fig. 1 The position of $q_{d}$ and $q_{1}$

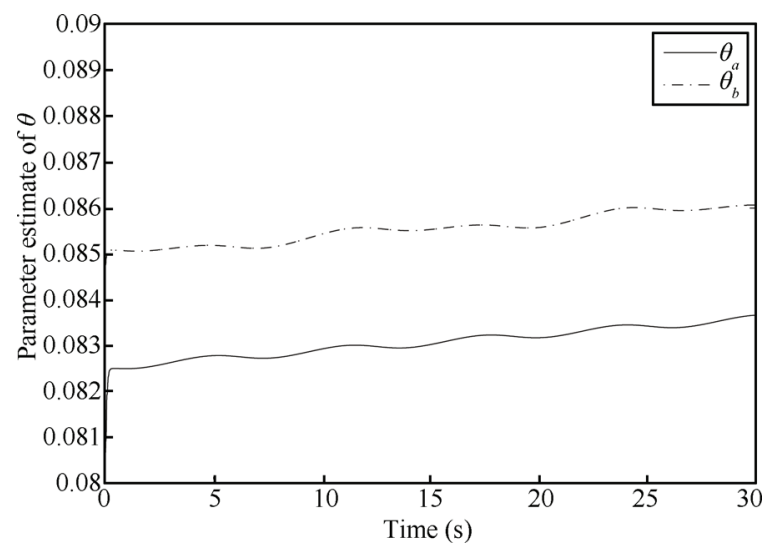

Fig. 2 The trajectories of $\hat{\theta}$

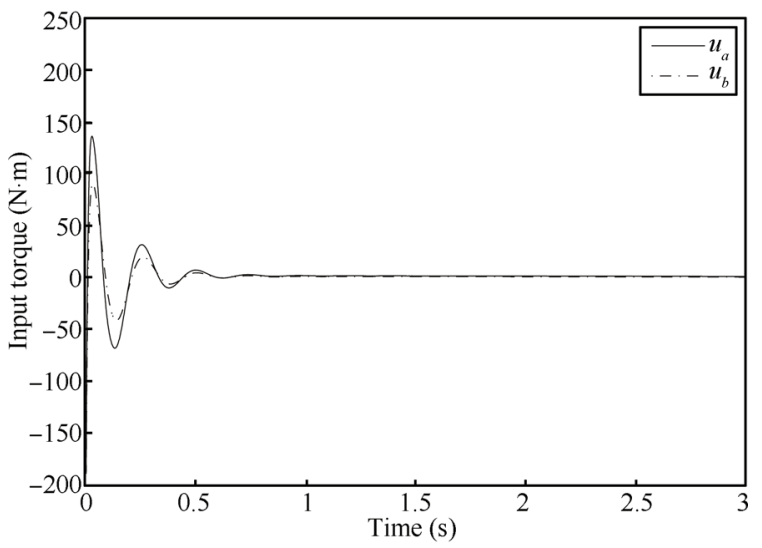

Fig. 3 The trajectories of input $u$

Since the responses of the presented controllers are similar, in this example, we only provide the simulation for the control method shown in Section 4. By (7), we have

$$
\left\{\begin{array}{l}
\dot{z}_{1}=z_{2}-\left(c_{1}+1\right) z_{1} \\
\dot{z}_{2}=I^{-1} K x_{3}-F\left(x_{1}, x_{2}, q_{d}, \dot{q}_{d}, \ddot{q}_{d}\right) \\
\dot{z}_{3}=x_{4}+\frac{1}{\tau_{3}} \varrho_{1} \\
\dot{z}_{4}=J^{-1}\left(u+B x_{4}+K\left(x_{1}-x_{3}\right)-f_{2}\right)+\frac{1}{\tau_{4}} \varrho_{2}
\end{array}\right.
$$

where $F=I^{-1}\left(M g l \sin x_{1}+K x_{1}+f_{1}\right)+\ddot{q}_{d}-\left(c_{1}+1\right) z_{2}+$ $\left(c_{1}+1\right)^{2} z_{1}$. We choose the first virtual control

$$
x_{3}^{*}\left(z_{1}, z_{2}, q_{d}, \dot{q}_{d}, \hat{\theta}\right)=-\left(\frac{1}{2}+c_{2}+\omega_{1} \hat{\theta}\right) z_{2}
$$

where $\omega_{1}=\frac{1}{\delta_{1}}\left(\sin ^{2} x_{1}+x_{1}^{2}\right)+\frac{1}{2 \delta_{2}}+c_{1}+\frac{\left(c_{1}+1\right)^{4}}{2}+2$. Let the virtual control $x_{3}^{*}$ pass the first-order filter (37), then we can get the filtered virtual control $\bar{x}_{3}$. Next, we choose the second virtual control

$$
x_{4}^{*}\left(z_{1}, z_{2}, z_{3}, q_{d}, \dot{q}_{d}, \hat{\theta}\right)=-\left(\frac{3}{2}+c_{3}\right) z_{3}-\frac{1}{\tau_{3}} \varrho_{1} .
$$

Let the virtual control $x_{4}^{*}$ pass the first-order filter (37), then we can get the filtered virtual control $\bar{x}_{4}$. At last, we choose the actual control and the adaptive law as

$$
\left\{\begin{array}{l}
u=-\left(\frac{1}{2}+\frac{1}{\epsilon_{3} \tau_{4}^{2}}+c_{4}+\omega_{2} \hat{\theta}\right) z_{4} \\
\dot{\hat{\theta}}=\gamma\left(\omega_{1} z_{2}^{\mathrm{T}} z_{2}+\omega_{2} z_{4}^{\mathrm{T}} z_{4}\right)-\sigma \hat{\theta}, \quad \hat{\theta}(0)=0.1
\end{array}\right.
$$

where $\omega_{2}=\frac{1}{2 \epsilon_{1}}\left|x_{1}-x_{3}\right|^{2}+\frac{1}{2 \epsilon_{2}} x_{4}^{2}$.

In the simulation, we choose the parameters as $c_{1}=1.5$, $c_{2}=3, c_{3}=2.5, c_{4}=3, \delta_{1}=0.005, \delta_{2}=0.04, \tau_{3}=0.001$, $\tau_{4}=0.03, \epsilon_{1}=0.1, \epsilon_{2}=0.01, \epsilon_{3}=8.5, \sigma=0.0004$ and $\gamma=$ 0.00055 . The initial values are $\bar{x}_{3}(0)=1.5$ and $\bar{x}_{3}(0)=3.5$. The simulation demonstrates that the tracking objective of flexible joint robots can be achieved with satisfactory responses by the designed controller (62).

\section{Concluding remarks}

Tracking problems of the FJR system which is underactuated, are more difficult than the fully actuated system. 
This paper gives a new adaptive tracking control method for uncertain flexible joint robots. The designed controllers can make the position tracking error arbitrarily small, while keeping all the closed signals globally/semi-globally uniformly ultimately bounded. In this direction, there are still remaining problems to be investigated. For example, an interesting research problem is how to design an adaptive tracking controller for the flexible joint robots in random vibration environment.

\section{Appendix}

Proof of Lemma 2. From Assumptions 1 and 2, we have

$$
\begin{gathered}
\left|M_{1}^{-1}\left(x_{1}\right)\left(C x_{2}+h\left(x_{1}\right)+K x_{1}+f_{1}\right)\right| \leq \\
\psi_{11}\left(x_{1}, x_{2}\right) \max \left\{\frac{\theta_{0}^{\frac{1}{2}}}{m_{1}}, \frac{\theta_{1}^{\frac{1}{2}}}{m_{1}}\right\}
\end{gathered}
$$

where $\psi_{11}\left(x_{1}, x_{2}\right)=\phi_{0}^{\frac{1}{2}}+\phi_{1}^{\frac{1}{2}}$. Then, we can deduce that

$\left|F_{1}\right| \leq \psi_{11} \bar{\theta}+\psi_{12}+\ddot{q}_{d}$.

From (8) and the definition of $F_{2}$ and $F_{3}$, it is not difficult to get that there exist smooth functions such that

$\left|F_{2}\right| \leq \psi_{21} \bar{\theta}+\psi_{22}+\psi_{23} \ddot{q}_{d}$
$\left|F_{3}\right| \leq \psi_{31} \bar{\theta}+\psi_{32}+\psi_{33} \ddot{q}_{d}$.

Thus, we can deduce that

$$
\begin{aligned}
& -z_{3}^{\mathrm{T}} F_{2} \leq z_{3}^{\mathrm{T}}\left(\psi_{21} \bar{\theta}+\psi_{22}+\psi_{23} \ddot{q}_{d}\right) \leq \\
& \quad\left(z_{3}^{\mathrm{T}} z_{3}\right)\left(\psi_{21}^{2} \theta+\psi_{22}^{2}+\psi_{23}^{2}\right)+\frac{\theta}{4}+\frac{1}{4}+\frac{\ddot{q}_{d}}{4}= \\
& \quad\left(z_{3}^{\mathrm{T}} z_{3}\right)\left(\psi_{1} \theta+\psi_{2}\right)+\nu
\end{aligned}
$$

where $\psi_{1}=\psi_{21}^{2}, \psi_{2}=\psi_{22}^{2}+\psi_{23}^{2}$ and $\nu=\frac{\theta}{4}+\frac{1}{4}+\frac{\ddot{q}_{d}}{4}$. Similarly, we can deduce that

$$
\begin{aligned}
& -z_{4}^{\mathrm{T}} F_{3} \leq z_{4}^{\mathrm{T}}\left(\psi_{31} \bar{\theta}+\psi_{32}+\psi_{33} \ddot{q}_{d}\right) \leq \\
& \quad\left(z_{4}^{\mathrm{T}} z_{4}\right)\left(\psi_{31}^{2} \theta+\psi_{32}^{2}+\psi_{33}^{2}\right)+\frac{\theta}{4}+\frac{1}{4}+\frac{\ddot{q}_{d}}{4}= \\
& \quad\left(z_{4}^{\mathrm{T}} z_{4}\right)\left(\psi_{3} \theta+\psi_{4}\right)+\nu
\end{aligned}
$$

where $\psi_{3}=\psi_{31}^{2}$ and $\psi_{4}=\psi_{32}^{2}+\psi_{33}^{2}$.

\section{References}

[1] S. Ozgoli, H. D. Taghirad. A survey on the control of flexible joint robots. Asian Journal of Control, vol. 8, no. 4, pp. 332$344,2006$.

[2] S. S. Ge. Adaptive controller design for flexible joint manipulators. Automatica, vol. 32, no. 2, pp. 273-278, 1996.

[3] A. Albu-Schäffer, C. Ott, G. Hirzinger. A unified passivitybased control framework for position, torque and impedance control of flexible joint robots. The International Journal of Robotics Research, vol. 26, no. 1, pp. 23-39, 2007.
[4] A. C. Huang, Y. C. Chen. Adaptive sliding control for single-link flexible-joint robot with mismatched uncertainties. IEEE Transactions on Control Systems Technology, vol. 12 , no. 5 , pp. $770-775,2004$.

[5] F. Abdollahi, H. A. Talebi, R. V. Patel. A stable neural network-based observer with application to flexiblejoint manipulators. IEEE Transactions on Neural Networks, vol. 17, no. 1, pp. 118-129, 2006.

[6] H. Chaoui, P. Sicard, W. Gueaieb. ANN-based adaptive control of robotic manipulators with friction and joint elasticity. IEEE Transactions on Industrial Electronics, vol. 56, no. 8, pp. 3174-3187, 2009.

[7] P. Tomei. Tracking control of flexible joint robots with uncertain parameters and disturbances. IEEE Transactions on Automatic Control, vol. 39, no. 5, pp. 1067-1072, 1994.

[8] W. Yim. Adaptive control of a flexible joint manipulator. In Proceedings of International Conference on Robotics and Automation, IEEE, Seoul, South Korea, pp. 3441-3446, 2001.

[9] M. S. Kim, J. S. Lee. Adaptive tracking control of flexiblejoint manipulators without overparametrization. Journal of Robotic Systems, vol. 21, no. 7, pp. 369-379, 2004.

[10] C. Liu, C. C. Cheah, J. J. E. Slotine. Adaptive task-space regulation of rigid-link flexible-joint robots with uncertain kinematics. Automatica, vol. 44, no. 7, pp. 1806-1814, 2008.

[11] T. P. Zhang, S. S. Ge. Adaptive dynamic surface control of nonlinear systems with unknown dead zone in pure feedback form. Automatica, vol. 44, no. 7, pp. 1895-1903, 2008.

[12] X. Y. Luo, Z. H. Zhu, X. P. Guan. Adaptive fuzzy dynamic surface control for uncertain nonlinear systems. International Journal of Automation and Computing, vol. 6 , no. 4, pp. 385-390, 2009.

[13] M. Z. Hou, G. R. Duan. Adaptive dynamic surface control for integrated missile guidance and autopilot. International Journal of Automation and Computing, vol. 8, no. 1, pp. 122-127, 2011.

[14] K. J. Yang, K. S. Hong, E. J. Rhee, W. S. Yoo. Model reference adaptive control of a flexible structure. KSME International Journal, vol. 15, no. 10, pp. 1356-1368, 2001.

[15] K. J. Yang, K. S. Hong, W. S. Yoo, F. Matsuno. Model reference adaptive control of a cantilevered flexible beam. JSME International Journal, Series C, vol. 46, no. 2, pp. 640-651, 2003.

[16] K. J. Yang, K. S. Hong, F. Matsuno. Robust adaptive control of a cantilevered flexible structure with spatiotemporally varying coefficients and bounded disturbance. JSME International Journal, Series C, vol.47, no. 3, pp.812-822, 2004 .

[17] D. Li, J. M. Li. Adaptive iterative learning control for nonlinearly parameterized systems with unknown time-varying delay and unknown control direction. International Journal of Automation and Computing, vol.9, no. 6, pp.578-586, 2012 . 
[18] B. S. Park, S. J. Yoo, J. B. Park, Y. H. Choi. A simple adaptive control approach for trajectory tracking of electrically driven nonholonomic mobile robots. IEEE Transactions on Control Systems Technology, vol.18, no. 5, pp.1199-1206, 2010.

[19] M. Y. Cui, Z. J. Wu, X. J. Xie, P. Shi. Modeling and adaptive tracking for a class of stochastic Lagrangian control systems. Automatica, vol. 49, no. 3, pp. 770-779, 2013.

[20] M. W. Spong. Modeling and control of elastic joint robots. Journal of Dynamic Systems, Measurement, and Control, vol. 109, no. 4, pp. 310-319, 1987.

[21] A. K. Kostarigka, Z. Doulgeri, G. A. Rovithakis. Prescribed performance tracking for flexible joint robots with unknown dynamics and variable elasticity. Automatica, vol. 49, no. 5 , pp. 1137-1147, 2013.

[22] K. D. Do, Z. P. Jiang, J. Pan. Robust adaptive path following of underactuated ships. Automatica, vol.40, no. 6, pp. 929-944, 2004.

[23] Z. J. Li, Y. N. Zhang. Robust adaptive motion/force control for wheeled inverted pendulums. Automatica, vol. 46, no. 8, pp. 1346-1353, 2010
[24] Y. F. Chen, A. C. Huang. Controller design for a class of underactuated mechanical systems. IET Control Theory and Applications, vol. 6, no. 1, pp. 103-110, 2012.

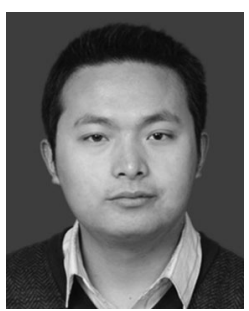

Zhen-Guo Liu received the M.Sc. degree from Qufu Normal University, China in 2012. He is currently a Ph. D. candidate in the School of Automation, Southeast University, China.

His research interests include nonlinear system control, adaptive theory and nonholonomic systems control.

E-mail: lzg819@163.com (Corresponding author)

ORCID iD: 0000-0003-1786-2400

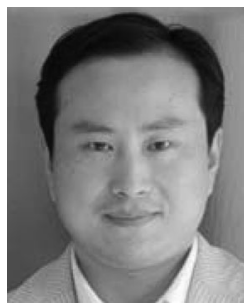

Jin-Ming Huang received the M. Sc. degree from Beijing Normal University, China in 2004. He is currently a Ph. D. candidate in the School of Engineering, Qufu Normal University, China. He is an associate professor at the Qufu Normal University, China.

His research interests include nonholonomic systems control, robotics, and nonlinear system control.

E-mail: huangjm_163@163.com 